\title{
Intrasound Therapy: An Effective Alternative to Sodium Diclofenac Phonophoresis in the Management of Mechanical Chronic Cervical Spine Pain
}

\author{
*AIYEGBUSI, AI; SANNI, HO; AKINBO, SR \\ ${ }^{I}$ Department of Physiotherapy, College of Medicine, University of Lagos, Nigeria, PMB 12003, Idiaraba, Lagos, Nigeria. 02341 \\ *Corresponding Author Email: aaiyegbusi@ unilag.edu.ng \\ Tel: +2348023212513
}

\begin{abstract}
The efficacy of Sodium Diclofenac Phonophoresis (SDP) as an effective adjunct in the management of inflammation and pain has been established though its application entails complicated choices of treatment parameters. Intrasound Therapy (IST), acclaimed for its simplicity of operation has been reported to promote healing though no studies have been done on its effect in Chronic cervical spine pain (CCSP). The aim of this study was to determine if IST could be an effective therapeutic option to SDP as an adjunct in the management of CCSP. Forty seven (47) participants with CCSP that had definite diagnoses were randomly assigned into 3 groups. All participants had exercises and massage while in addition, group 1 had SDP and group 2 IST for 10 minutes each. Participants were treated for 40 minutes twice a week for 4 weeks and were evaluated for pain, Quality of life $(\mathrm{QoL})$, disability and range of motion (ROM) of the cervical spine. Paired sample t-test was used to compare the outcome parameters in each group and data presented as Mean \pm SEM with significance at $\mathrm{p}<0.05$. IST and SDP significantly $(p<0.05)$ improved the clinical parametres compared with the control group and there were no significant $(p>0.05)$ differences in clinical outcome between the IST and SDP groups. IST was as effective as SDP and considering its relative simplicity of operation could be an alternative therapeutic adjunct in the management of chronic cervical pain.
\end{abstract}

\section{DOI: https://dx.doi.org/10.4314/jasem.v23i2.26}

Copyright: Copyright (C) 2019Aiyegbusi et al. This is an open access article distributed under the Creative Commons Attribution License (CCL), which permits unrestricted use, distribution, and reproduction in any medium, provided the original work is properly cited.

Dates: Received: 17 November 2018; Revised: 19 January 2019; Accepted 22 January 2019

Keywords: Sodium Diclofenac Phonophoresis, Intrasound therapy, Chronic Neck pain, Neck Disability

Chronic neck pain is a highly prevalent condition with about two-thirds of the adult population affected at some point in their lives (Brian et al, 2016). ${ }^{[1]}$ Chronic neck pain is increasingly recognized as one of the most common causes for disability (Chartered Society of Physiotherapy, 2015). It has extensive diagnoses; the most common causes being biomechanical, such as axial neck pain, whiplash-associated disorder and cervical radiculopathy (Douglas and Bope, 2004) ${ }^{[4]} \mathrm{A}$ previous study suggested that combined physiotherapy treatment including active and passive modalities is effective for improvement in pain intensity, disability, and quality of life (QoL) in participants with mechanical chronic neck pain (Seyda et al, 2016).$^{[5]}$ The treatment goals are to relieve pain, reduce muscle spasm, improve range of motion and muscle strength, correct postural abnormalities and ultimately improve functional status and QoL (Philadelphia panel, 2001). ${ }^{[6]}$ Therapeutic Ultrasound (TUS) has been widely used in the treatment of musculoskeletal disorders including Phonophoresis (PP) which is generally defined as driving a topically applied medication through the skin with TUS as a medium. ${ }^{[8]} \mathrm{PP}$ has been used to enhance transdermal drug delivery in sports medicine and orthopaedic rehabilitation and is believed to accelerate functional recovery by decreasing pain and promoting healing through the activation of fibroblast activity with collagen deposition (Maria Jose, 2015). A systematic review on the management of patients with whiplashassociated disorders or neck pain and associated disorders had concluded that some passive physical modalities such as heat, cold, diathermy, hydrotherapy, and ultrasound are not effective and should not be used to manage neck pain, even as another study observed that PP with capsaicin treatment was effective in the treatment of patients with CNP and thus suggested that a combination of PP with exercises can be used to obtain optimal clinical results (Dumus at al, 2014; Wong, 2016). There are however a number of limitations to the use of PP including high cost of the TUS machine, the complexity of operation due to the wide variables in treatment parameters, the cumbersome nature of the machine and the problem of nanobubbles/nanodroplets which inhibits optimal perfusion of drugs via the skin to target tissues (YingZheng et al, 2013). Studies on the efficacy of TUS demonstrate that there are no precise guidelines for its parameters, particularly with respect to the dose- 
response of the treatments which is influenced by many variables, including frequencies, intensities, irradiation times, application modes, type and coupling techniques, and early post-injury interventions (Warden and McMeeken, 2002; Fu et al, 2008). These factors become pertinent when compared with the intrasound device which has the advantage of being a simple, non-invasive, relatively affordable and easily operated device that has been documented in literatures to be an effective adjunct in the treatment of some musculoskeletal disorders especially in the management of knee osteoarthritis (Aiyegbusi et al, 2015). The Intrasound device produces mix frequency acoustic waves in the intrasonic range $(16,000-20,000 \mathrm{~Hz})$ with claims of its efficacy in the management of a wide range of ailments, including improved healing in acute inflammatory injuries especially in tendon injuries when used on animal models (Dancocks et al, 1997; Aiyegbusi et al, 2010). The purpose of this study was therefore to investigate the comparative therapeutic efficacy of SDP and IST in improving ROM, pain, QoL and reduction in disability levels associated with CCSP in 47 participants treated twice weekly for 4 consecutive weeks.

\section{MATERIALS AND METHODS}

Subject Selection: Forty seven (47) participants (20 males and 27 females) with chronic neck pain that had definite diagnoses like axial neck pain, whiplash associated disorder and cervical spondylosis with or without radiculopathy were recruited at the physiotherapy outpatient clinic of a tertiary hospital. The participants were assessed by obtaining a detailed history and by carrying out physical examination and radiographic assessment. They gave duly signed written informed consent and approval was sought and obtained from the institution Health Research and Ethics Committee (Approval Number: ADM/DCST/HREC/APP/763) prior to the commencement of the study. Excluded from the study were participants with severe chronic neck pain (using the Neck Disability Index Scores), cognitive limitations and those who have had surgery of the cervical or upper thoracic spine. A non-probability consecutive sampling technique was employed in recruiting the participants that met the inclusion criteria as they became available between April and September 2016. Eligible participants were then randomly assigned into the study groups by a blinded research assistant who had no knowledge of the group allocations using concealed randomly generated group allocation numbers in a bowl. All the eligible participants were neither on any medication nor any form of co-intervention during the period of study. The sample size was estimated to be 46 using the statistical formula by Cohen (1992).

Participants' Grouping: Group 1: (SDP group) had participants who were treated using massage, isometric exercises and SDP. Group 2: (IST group) participants received massage, isometric exercises and IST

Group 3: (Control group) participants received massage and isometric exercises only

Materials: The Intrasound Device used was the Professional Novasonic Sonic Wave SK2 device, made in USA while the Therapeutic Ultrasound Machine was US-2000 $2^{\text {nd }}$ edition portable ultrasound therapy machine, made in USA and it was used for the Phonophoresis therapy.

Therapeutic Intervention: Group 1 (SDP group) participants were treated using massage, isometric exercises and SDP, Group 2 (IST group) participants received massage, isometric exercises and IST while group 3 participants (Control group) were treated using massage and isometric exercises only.

Prior to the commencement of the intervention, all the participants were assessed and evaluated for pain intensity using Numeric Pain Rating Scale (NPRS) (Rodriguez, 2001) and the disability level using the Neck Disability Index Questionnaire (NDI) which is a self-report questionnaire that has been shown to demonstrate a high degree of test-retest reliability (ICC=0.91) and internal consistency (Vernon, 1996; Aslan et al, 2008). The health-related quality of life was evaluated with the Short-Form 36 Health Survey (SF-36) that includes 8 health domains with scores in each category that range from 0 to 100 ; higher scores indicating a better QoL (Ware and Sherbourne, 1992). The goniometry for head rotation, neck extension and flexion and neck side flexion were performed and read to the nearest degree using a Goniometer (G 300 Model) (Hachadorian et al, 2010). Pain and quality of life were evaluated as primary outcomes while the neck disability and neck range of motion were the secondary outcome measures.

Treatment Protocol: The participants went through the following sequence of treatment regimen:

Group 1: The sodium diclofenac gel was applied over the surface area of the neck to be treated at a dosage of 2 Finger Tip Unit (FTU) which is equal to 1 gram (Long and Finlay, 1991). TUS was applied over the cervical and upper thoracic spine with the treatment head in circular motion at medium intensity for 10 minutes. The participants afterwards underwent the same exercise protocol as those in the control group. 
Group 2: The acoustic gel (which is of no pharmacological significance) was applied over the surface of the neck to be treated at a dosage of 2 Finger Tip Unit (FTU) (Long and Finlay, 1991). The treatment head of the intrasound device was applied in circular motion over the cervical and upper thoracic spine for 10 minutes. The control knob was adjusted to an intensity the patient was comfortable with (Aiyegbusi et al, 2010). The participants afterwards underwent the same exercise protocol as those in the control group.

Group 3 (Control Group): The intervention consisted of a selection of soft tissue massage, muscle energy techniques, resistance exercises and manual cervical traction applied to the neck region. Every session of treatment included soft tissue massage which was applied using cross-fibre kneading, longitudinal stretch and inhibition to the cervical and sub-occipital muscles for 5 minutes (Cen et al, 2003) followed by muscle energy technique which included isometric exercises given in all the range of motion to facilitate stretching of the neck muscles for 5 minutes (Chaitow, 1996). Resistance was manually applied in all the range of motion of the cervical region (10-repititions, 3-bouts) to strengthen the muscles around the neck region. Postural advice and exercise prescription of neck mobility and stretching were included as part of the home programme. Manual cervical traction was given for 5 minutes with the traction force applied manually to the cervical region and a rotational range of motion carried out within the limit of pain, on both sides. (Kruse et al, 2000).

Data Analysis: Statistical Package for Social Science (SPSS Inc., Chicago, Illinois, USA) 21.0 version for Windows package program was used to analyze data. Demographic and quantitative data was expressed as Mean \pm Standard Error of Mean (SEM) and confidence interval $(\mathrm{CI})$, to describe the differences in related treatments. Paired sample t-test was used to compare the baseline/pre-treatment and post-treatment variations in outcome variables in each group. ANOVA was used to determine significant differences in the changes between the three groups with a further post-hoc analysis.

\section{RESULTS AND DISCUSSION}

Forty seven participants were recruited but a total of thirty-seven (37) participants; 15 males and 22 females with a mean age of $53.2 \pm 1.81$ years completed the study. Comparison of baseline values of the outcome parameters using analysis of variance (ANOVA) showed no significant differences except for right and left side flexion which were significantly higher in group $1 \quad(p<0.05)$. Application of IST resulted in significant $(\mathrm{p}<0.05)$ improvement in all the primary and secondary outcome parameters while in the other two groups there was significant $(\mathrm{p}<0.05)$ impact on most of the domains (tables $1 \& 2$ ). As seen in tables 3 , the mean changes in some of the outcome parameters between the groups were significant $(\mathrm{p}<0.05)$ in some of the domains.

The marked improvement in clinical outcomes seen in all the groups underscores the clinical efficacy of the interventions given. The Control Group showed significant $(\mathrm{p}<0.05)$ improvement in most of the clinical outcome parameters and this corroborates the report of Seydar et al,(2016) which suggested the efficacy of stabilization exercises with connective tissue massage over stabilization exercises alone. This finding is also in synchrony with results from a prior study where manual therapy was used to treat both acute and chronic neck pain and it was suggested that the use of manipulation and/or mobilization was a viable option for the management of neck pain though from our results, the clinical impact of these exercises on the disability index was minimal (Cabak, 2005). The significant improvement seen in the clinical outcome measures for the SDP group is in conformity with previous studies that had established the therapeutic efficacy of various types of NSAIDs gel phonophoresis on both acute and chronic musculoskeletal pain and disorders (Akinbo et al, 2011).

The effects of SDP have been attributed to its ability to enhance the penetration of sodium diclofenac into the tissues thereby resulting in therapeutic benefits (Cross et al, 1998). There are however a number of issues with the application of SDP, one of which is the wide and complicated choices of treatment parameters of TUS which makes it difficult to get consistent results from different studies (Warden and McMeeken, 2002). The intrasound device on the other hand has the advantage of being simple and noninvasive with ease of operation. In this study, IST significantly improved the outcome parameters in all the clinical domains and with superior significant differences in the mean changes when compared to those in the control group. This further provides a support for a prior study that concluded that compared with TENS, intrasound therapy is an effective adjunct in the management of OA knee (Aiyegbusi et al, 2015). 
Table 1: Analysis of primary clinical outcome measures of patients in the three groups, pre- and post-intervention

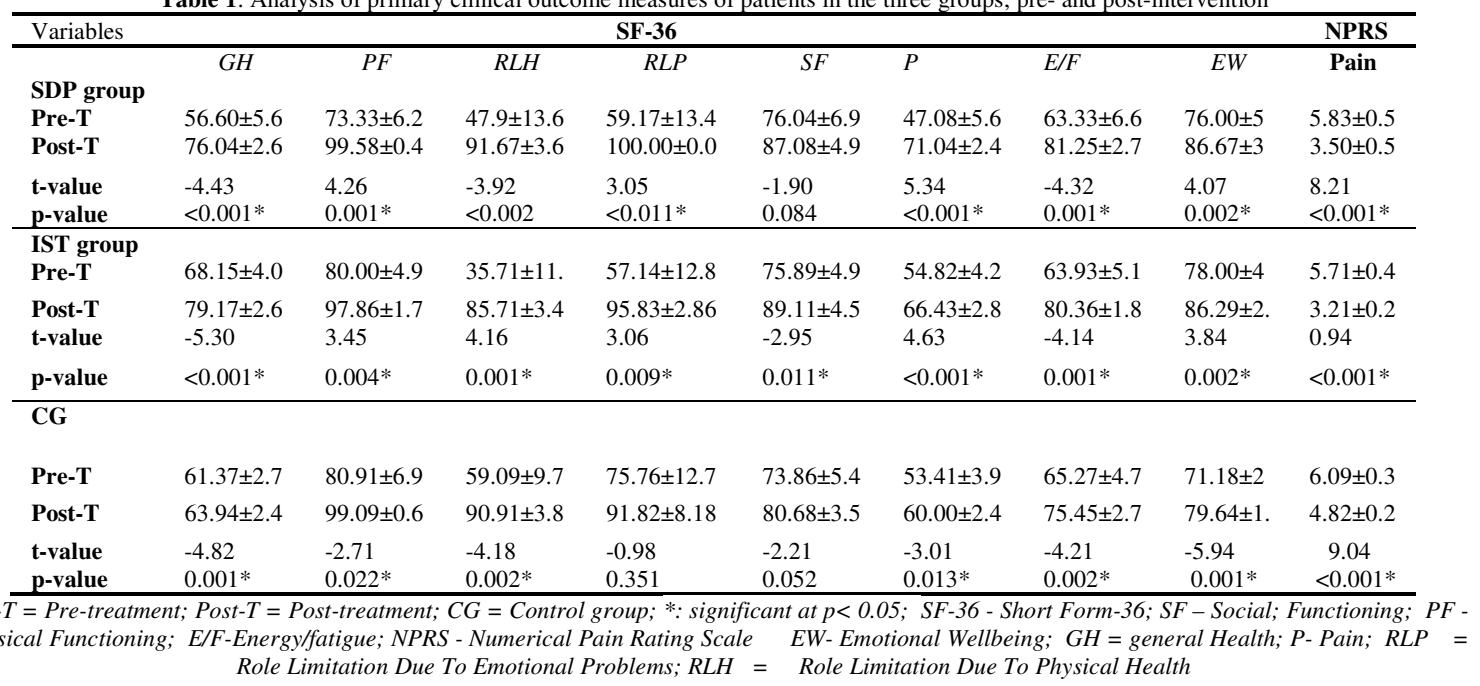

Table 2: Analysis of secondary clinical outcome measures of patients in the three groups; Pre- and Post-intervention

\begin{tabular}{|c|c|c|c|c|c|c|c|}
\hline \multirow[t]{2}{*}{ Variables } & \multicolumn{6}{|c|}{ RANGE OF MOVEMENT } & \multirow{2}{*}{$\frac{\text { NDI }}{\text { NDI }}$} \\
\hline & Flex & Ext & $S F R$ & $S F L$ & $R R T$ & $R L T$ & \\
\hline SDP group & & & & & & & \\
\hline Pre-T & $33.75 \pm 3.3$ & $38.75 \pm 3.4$ & $36.25 \pm 3.15$ & $34.58 \pm 2.5$ & $55.83 \pm 2.8$ & $55.00 \pm 2.7$ & $10.92 \pm 2.09$ \\
\hline Post-T & $42.50 \pm 1.9$ & $50.00 \pm 1.8$ & $41.67 \pm 1.42$ & $40.83 \pm 1.4$ & $64.17 \pm 2.2$ & $64.58 \pm 1.6$ & $3.92 \pm 1.22$ \\
\hline t-value & 4.99 & -4.18 & 2.72 & -4.10 & 5.38 & -6.67 & 6.28 \\
\hline p-value & $<0.001 *$ & $0.002 *$ & $<0.020^{*}$ & $<0.002 *$ & $<0.001^{*}$ & $<0.001 *$ & $<0.001 *$ \\
\hline IST group & & & & & & & \\
\hline Pre-T & $30.36 \pm 2.4$ & $35.00 \pm 2.5$ & $32.50 \pm 2.66$ & $30.71 \pm 2.8$ & $55.00 \pm 2$ & $54.29 \pm 2.9$ & $16.00 \pm 5.95$ \\
\hline Post-T & $37.50 \pm 1.6$ & $44.29 \pm 1.9$ & $37.50 \pm 1.80$ & $38.57 \pm 1.6$ & $60.00 \pm 1.7$ & $59.64 \pm 2.2$ & $3.00 \pm 0.671$ \\
\hline t-value & 7.07 & -6.32 & 2.75 & -4.38 & 2.75 & -3.32 & -2.21 \\
\hline p-value & $<0.001 *$ & $0.001 *$ & $0.016^{*}$ & $0.001 *$ & $0.016 *$ & $<0.006^{*}$ & $<0.046^{*}$ \\
\hline
\end{tabular}

\begin{tabular}{llllllll}
\hline CG & \multicolumn{7}{c}{} \\
Pre-T & $38.64 \pm 3.4$ & $32.73 \pm 3.5$ & $25.00 \pm 1.78$ & $23.64 \pm 2.3$ & $54.09 \pm 2.6$ & $52.27 \pm 1.8$ & $7.00 \pm 0.71$ \\
Post-T & $42.73 \pm 2.2$ & $40.00 \pm 2.3$ & $36.36 \pm 1.80$ & $37.73 \pm 1.6$ & $60.91 \pm 0.6$ & $60.00 \pm 1.3$ & $6.09 \pm 0.63$ \\
t-value & 3.19 & -2.76 & -2.67 & -0.959 & -7.04 & -2.89 & -3.56 \\
p-value & $0.010^{*}$ & $0.020^{*}$ & $0.024^{*}$ & $<0.001^{*}$ & $<0.001^{*}$ & $0.016^{*}$ & $<0.005$
\end{tabular}

*: significant at $p<0.05$; Key: SFR-Side Flexion Right SFL-Side Flexion Left; RRT-Rotation Right; RLT-Rotation Left; Flex- Flexion; Ext-Extension; NDI - Neck Disability Index

As presented in table 3, where the mean differences in the outcome parameters between the groups were analyzed, IST compared with SDP had better clinical effects over the control group in many of the domains. These results further substantiate the claims that intrasound therapy helps to revive and support the selfhealing power of the body through gentle, improvement in tissue nutrition at a cellular level thus stimulating the natural healing process of the body (Novasonic, 2014).

This is particularly important as it has been earlier suggested that rather than just pain relief, pain management should involve setting a course for the healing of the tissues that are causing the pain (Barker and Meletis, 2004). It is pertinent to note that a prior study had concluded that the higher the frequency of treatment, the better the outcome and it was reported that twice daily treatment with IST resulted in better healing than the once daily treatment (Aiyegbusi et al, 2015).

In this study, IST was given for 10 minutes twice a week as an adjunct to exercises, traction and massage yet the clinical effect on mechanical neck pain was quite significant. As a follow-up to this study, it will therefore be important to determine the effect of IST when given either once or twice daily rather than twice weekly. 
Table 3: Comparative analysis of the mean changes in outcome parameters between the groups

\begin{tabular}{|c|c|c|c|c|c|}
\hline Groups & $\begin{array}{l}1 / 2 \\
\mathrm{MD} \pm \mathrm{SEM}\end{array}$ & $\begin{array}{l}2 / 3 \\
\mathrm{MD} \pm \mathrm{SEM}\end{array}$ & $\begin{array}{l}1 / 3 \\
\text { MD } \pm \text { SEM }\end{array}$ & F-value & $p$-value \\
\hline \multicolumn{6}{|c|}{ Primary Outcome Measure } \\
\hline \multirow{2}{*}{\multicolumn{6}{|c|}{ SF-36: }} \\
\hline & & & & & \\
\hline $\mathrm{GH}$ & $2.11 \pm 2.51$ & $9.27 \pm 2.55^{\dagger}$ & $7.14 \pm 2.48^{\dagger}$ & 7.35 & $0.002 *$ \\
\hline $\mathrm{PF}$ & $1.86 \pm 1.75$ & $0.65 \pm 1.86$ & $-1.22 \pm 1.78$ & 0.59 & 0.559 \\
\hline RLH & $4.25 \pm 4.48$ & $2.32 \pm 4.75$ & $-1.93 \pm 4.68$ & 0.45 & 0.642 \\
\hline RLP & $4.20 \pm 6.44$ & $7.93 \pm 6.91$ & $3.74 \pm 6.69$ & 0.66 & 0.522 \\
\hline SF & $-2.09 \pm 4.94$ & $5.38 \pm 0.94$ & $7.47 \pm 5.07$ & 1.12 & 0.337 \\
\hline $\mathrm{P}$ & $7.82 \pm 2.46^{\dagger}$ & $13.66 \pm 2.59^{\dagger}$ & $5.84 \pm 2.47$ & 14.08 & $0.000 *$ \\
\hline $\mathrm{E} / \mathrm{F}$ & $1.12 \pm 1.71$ & $6.53 \pm 1.82^{\dagger}$ & $5.41 \pm 1.76^{\dagger}$ & 7.35 & $0.002 *$ \\
\hline EW & $1.38 \pm 1.40$ & $4.62 \pm 1.50^{\dagger}$ & $3.25 \pm 1.47$ & 4.93 & $0.013 *$ \\
\hline \multicolumn{6}{|c|}{ Secondary Outcome Measure } \\
\hline NDI & $1.25 \pm 1.18$ & $-3.68 \pm 1.24^{\dagger}$ & $-2.43 \pm 1.25$ & 4.49 & $0.019 *$ \\
\hline \multicolumn{6}{|l|}{ ROM: } \\
\hline Neck Flex & $2.99 \pm 0.99^{\ddagger}$ & $2.67 \pm 1.06^{\dagger}$ & $-0.32 \pm 1.05$ & 5.38 & $0.009^{*}$ \\
\hline Neck Ext & $4.04 \pm 2.12$ & $7.31 \pm 2.28^{\dagger}$ & $3.27 \pm 2.16$ & 5.16 & $0.011 *$ \\
\hline Side Flex (Rt) & $2.24 \pm 1.56$ & $-0.19 \pm 1.83$ & $-2.53 \pm 1.67$ & 1.66 & 0.206 \\
\hline Side Flex (Lt) & $0.56 \pm 1.42$ & $-1.71 \pm 1.65$ & $-2.27 \pm 1.51$ & 1.15 & 0.329 \\
\hline Rotation (Rt) & $3.79 \pm 1.56$ & $2.46 \pm 1.66$ & $-1.32 \pm 1.60$ & 3.00 & 0.064 \\
\hline Rotation (Lt) & $4.56 \pm 1.74^{\dagger}$ & $3.11 \pm 1.86$ & $-1.44 \pm 1.79$ & 3.49 & $0.042 *$ \\
\hline
\end{tabular}

*: significant at $p<0.05 ;{ }^{\prime}$ significant changes between groups; SF-36 = Short Form-36; EW = Emotional Wellbeing; ROM = Range Of Motion; $E / F=$ Energy/fatigue $; N P R S=$ Numerical Pain Rating Scale $;$ NDI = Neck Disability Index $;$ EXT = Extension; RLP = Role Limitation Due To

Emotional Problems; $R L H=$ Role Limitation Due To Physical Health; SF = Social Functioning; $P=$ Physical Functioning; $P=$ Pain $;$ GH $=$ General Health; Gpl=SDP Gp2=IST Gp3=Control

In view of the many issues with the use of SDP including the complexity of operation of TUS and the problem of nanobubbles/nanodroplets (Ying-Zheng et al, 2013), we report that IST could be an effective and better adjunct over SDP in the management of CCP considering its simplicity of operation without complicated choices of treatment parameters as seen in TUS.

Conclusion: It is concluded from these findings that IST was as effective as SDP and considering its relative simplicity of operation could be an alternative therapeutic adjunct in the management of chronic cervical pain. One limitation of this study was the small sample size and the large number of drop-outs due to logistic reasons. Further studies are recommended with larger sample sizes and increased frequency of treatment sessions.

\section{REFERENCES}

Aiyegbusi, AI; Duru, FIO; Akinbo, SR; Noronha, CC; Okanlawon, AO (2010). Intrasound therapy in tendon healing: Is intensity a factor? Open Access J of Rheumat: Res \& Rev.2:45-52

Aiyegbusi, AI; Okafor, UAC; Adejugbagbe, MK; Akinfeleye, A; Fapojuwo, OA (2015). Intrasound therapy is an effective adjunct in the Management of Osteoarthritis of the Knee: A randomized control trial. Clini Med Rev in Therapeutics. 7:1117.
Akinbo,SR; Owoeye, OB; Adesegun, S (2011). Comparison of the therapeutic efficacy of diclophenac sodium and methyl salicylate phonophoresis in the management of knee osteoarthritis. Turkish J of Rheumat. 26(2):111119.

Aslan, E; Karaduman, A; Yakut, Y, Aras, B; Smsek, IE, Yagl, N (2008). The cultural adaptation, reliability and validity of neck disability index in patients with neck pain: a Turkish version study. $\mathrm{J}$ of Spine. 33:E362-5.

Barker,JE; Meletis, CD (2004). Naturopathic Pain Management. Alternt \& Complem Therap. 10(4):188-193.

Brian, PC; Anuj, S; Troy, M (2016). Neck Pain in Adults: in Differential Diagnosis in Spine Surgery by John D Koerner, Daniel H Kim Jaypee Brothers Medical Publishers Ltd. New Delhi. 10:138.

Cabak, A; Maczewska, M; Lyp, M; Dobosz, J; Gasiorowoska, U (2005). The effectiveness of phonophoresis with ketoprofen in the treatment of epicondylopathy. Orthop Traumat Rehab. 7:6605.

Cen, SY; Loy, SF; Sletten, EG; Mclaine, A (2003). The effect of Traditional Chinese Therapeutic 
Massage on individuals with neck pain. Brit Med J. 4:88-93.

Chaitow, L (1996). Muscle energy techniques. Edinburgh: Churchill Livingstone. Chartered Society of Physiotherapy (2015). A physiotherapy clinical pathway for the Management of Chronic Neck Pain. DOI: http://dx.doi.org/10.1016/j.physio.03.1479

Cohen, J (1992) . A power primer psychological bulletin. 112(11): 155-159.

Cross, SE; Anderson, C; Roberts, MS (1998). Topical penetration of commercial salicylate esters and salts using human isolated skin and clinical microdialysis studies. Brit $\mathbf{J}$ of Clinic Pharmac. 46:29-35.

Dancocks, A; Rouse, A; Hiscox, J (1997). A pilot study to assess the sensitivity and specificity of an intrasound device in the diagnosis of ankle fracture. J Acc and Emerg Med. 14:230-232.

Douglass, AB; Bope, ET (2004). Evaluation and treatment of posterior neck pain in family practice. J of Am Board of Fam Pract. 17:13-22.

Durmus,D; Alayli, G; Tufekci, T; Kuru, O (2014). A randomized placebo-controlled clinical trial of phonophoresis for the treatment of chronic neck pain. Rheumatol Int. 34(5):605-11.

Fu, SC; Shum, WT; Hung, LK. (2008). Low-intensity pulsed ultrasound on tendon healing: a study of the effect of treatment duration and treatment initiation. Amer J of Sports Med. 36:1742 - 1749.

Hachadorian, J; Lugo, A; Lian, E; Truong, QDK; Vo, VT (2010). Measurement of range of neck motion: A comparative study. The Third International conference on the Development of Biomedical Engineering in Vietnam. 2010 http://dx.doi.org/10.1007/978-3-642-12020-6_35

Kruse, RA; Lmbarlina, F; De Bono, VF (2001). Treatment of cervical radiculopathy with flexion distraction. J of Manip Physiol Therapy. 24:206209.

Long, CC ; Finlay, AY (1991). The finger-tip unit- a new practical measure. Clinic and Exper Dermat. 16:444-447.

Maria José, MS; Morsoleto, FMS; Morsoleto, AP; Alves de Matos, FMR; Morgadoand, CAAA (2015). A Study of the Effects of Phonophoretic
Application of Hydrocortisone on the Regeneration of Injuries to Skeletal Muscle. Microsc. Microanal. 21 (Suppl 5): 77-78

Novasonic. How Novasonic-Novafon massager works. Available at: http://www. novasonicwest.com/ novasonic.asp. Accessed on October 06, 2014.

Philadelphia Panel (2001). Philadelphia panel evidence-based clinical practice guidelines on selected rehabilitation interventions for neck pain. Journal of Physical Therapy. 81:1701-16.

Rodriguez, CS (2001). Pain measurement in the elderly: a review. Pain Manag Nurs. 2:38-46.

Seyda, TC; Derya, OK; Turkan, A (2016). Cervical and scapulothoracic stabilization exercises with and without connective tissue massage for chronic mechanical neck pain: A prospective, randomized controlled trial. Musculosk Sci \& Pract. 21:144150

Uhlemann, C; Heinig, B; Wollina, U (2003). Therapeutic ultrasound in lower extremity wound management. The Int $\mathbf{J}$ of lower Extremity Wounds. 2(3): 152-7.

Vernon, H (1996). The neck disability index: Patient assessment and outcome monitoring in whiplash. J Musculosk Pain. 4(4):95-104.

Warden,SJ; McMeeken, JM (2002). Ultrasound usage and dosage in sports physiotherapy. Ultra Med Biol. 28(8):1075-80.

Ware, JE Jr; Sherbourne; CD (1992). The MOS 36item short-form health survey (SF-36). I. Conceptual framework and item selection. Med Care. 30 (6):473-83.

Wong, JJ; Shearer, HM; Mior, S; Jacobs, C; Côté, P; Randhawa, K (2016). Are manual therapies, passive physical modalities, or acupuncture effective for the management of patients with whiplash-associated disorders or neck pain and associated disorders? An update of the Bone and Joint Decade Task Force on Neck Pain and Its Associated Disorders by the OPTIMa collaboration. Spine J_16(12):1598-1630.

Ying-Zheng, Z; Li-Na,D; Cul-Tao, L; Yi-Guang, J; Shu-Ping, G (2013). Potential and problems in intrasound-responsive drug delivery systems. Intern J of Nano Medicine. 8:1621-1633. 